\title{
Upstream ULF waves and energetic electrons associated with the lunar wake: Detection of precursor activity
}

\author{
W. M. Farrell ${ }^{1}$, R.J. Fitzenreiter ${ }^{1}$, C. J. Owen ${ }^{2}$, J. B.

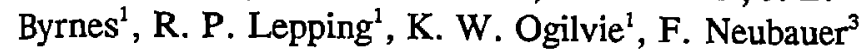

Abstract. We present observations of precursor ULF wave activity and energetic electron flows detected by the WIND spacecraft just prior to entry of the lunar wake on 27 December 1994. This activity occurs upstream of the wake on field lines directly connected to the wake penumbra region. The activity ceases near the penumbra entrance. The observations of upstream ULF wave activity and solar wind counterstreaming electron flows is similar to observations made upstream of collisionless bow shocks. Analogously, the wake precursor region is characterized by thermalization and information propagation ahead of the wake structure.

\section{Introduction}

The WIND spacecraft was launched on 1 November 1994 as a laboratory to study the normal and disturbed solar wind magnetoplasma prior to reaching the earth. In order to achieve a favorable orbital trajectory, the WIND spacecraft made a close swingby of the moon on 27 December 1994. Although not a primary mission objective, this close lunar encounter allowed an opportunity to study the interaction of the large body with the solar wind, now with unprecedented temporal, spatial, and spectral resolution. As described in the previous paper, a distinct observation of a lunar wake was made by the WIND magnetometer (MFI) lOwen et al., 1996]. In this paper we report on activity occurring in a region adjacent to the wake. As we shall describe, the nature of the activity bares a striking resemblance to activity in foreshock regions commonly observed adjacent to planetary and interplanetary shocks.

As magnetoplasma flows around a large obstacle like the earth's moon, both ions and electrons trajectories are dramatically affected. As described by Ness [1970] and references therein, botl species are absorbed at the lunar surface, thereby creating a void of plasma in the trailing lunar wake region. By removing the plasma, the perturbing body also creates a magnetic wave that extends outward from belind the moon, much like the wave associated with a boat wake. The size of the lunar radius is much larger than the ion gyroradius, and as such both solar wind electron and ion trajectories are altered by the occluding lunar disk. Consequently, the magnetic wake disturbance associated with the moon is considered Alfvenic in nature. Due to their greater thermal speed, the ambient electrons fill in the evacuated wake region faster than the ions, thereby creating an ambipolar electric field that retards the velocities of electrons and increases the velocities of the ions in a self-consistent way. In theory, such an electric field may be detected some distance upstream from the wake region by the reflected particles and corresponding wave signature.

In the late 60 's, a number of scientific studies focused on the wake in the solar wind formed by the moon. Specifically, a clear and repeated

\footnotetext{
'Laboratory for Extraterrestrial Physics, NASA/Goddard Space Flight Center, Greenbelt, MD 20771.

${ }^{2}$ Hughes/STX, NASA/Goddard Space, Flight Center, Greenbelt, MD 20771.

${ }^{3}$ Institut fur Geophysics und Meteorlogie der Universitat zu Koln, 500 Koln-41, Germany.
}

Copyright 1996 by the American Geophysical Union.

Paper number 96GL01355

0094-8534/96/96GL-01355\$05.00 identification of a lunar wake was made by Explorer 35 as it orbited close ( $<5$ lunar radii) to the moon (again, see the review by Ness [1970] and references therein). As detected by the onboard magnetometer, the edges of the wake consist of a distinct magnetic compression followed by a longer rarefaction. In the central wake region, the magnetic field strengths tend to rise due to the collective effects of currents generated at the wake boundary [Owen et al., 1996]. The signature of the lunar wake is quite clear, with these compression/rarefaction regions being located symmetrically about the central wake region. In many instances, the rarefactions are more distinct than the compressions, thus these features are more easily recognizable in a time-series [Whang and Ness, 1970].

Besides the quasi-DC magnetic signature of the lunar wake, Explorer 35 also detected significant ULF wave activity both upstream and downstream of the wake, on field lines magnetically-connected to the penumbral region [Ness and Schatten, 1969]. Close to the moon, the signal level of the waves became significant, approaching 2-3 nanoTesla (nT) in total magnitude. Thus, the wave variation (i.e., Delta-B/B) approached $50 \%$. Unfortunately, the spectral properties of the waves were difficult to determine due to a mismatch between the sampling rate of $0.2 \mathrm{~Hz}$ and the intrinsic sensor bandwidth of $5 \mathrm{~Hz}$ [Ness and Schatten, 1969]. Associated particle distribution measurements were also unavailable. It was postulated that electron anisotropies [Ness and Schatten, 1969] or perturbed electron flows from gradient drifts [Krall and Tidman, 1969] were responsible for the upstream wave activity, but clearly more details with respect to the wave and electron properties were required to make any definitive statement.

It has been nearly 25 years since a spacecraft with proper instrumentation passed close enough to the lunar wake to make scientific studies. On 27 December 1994, the WIND spacecraft passed within 7 lunar radii

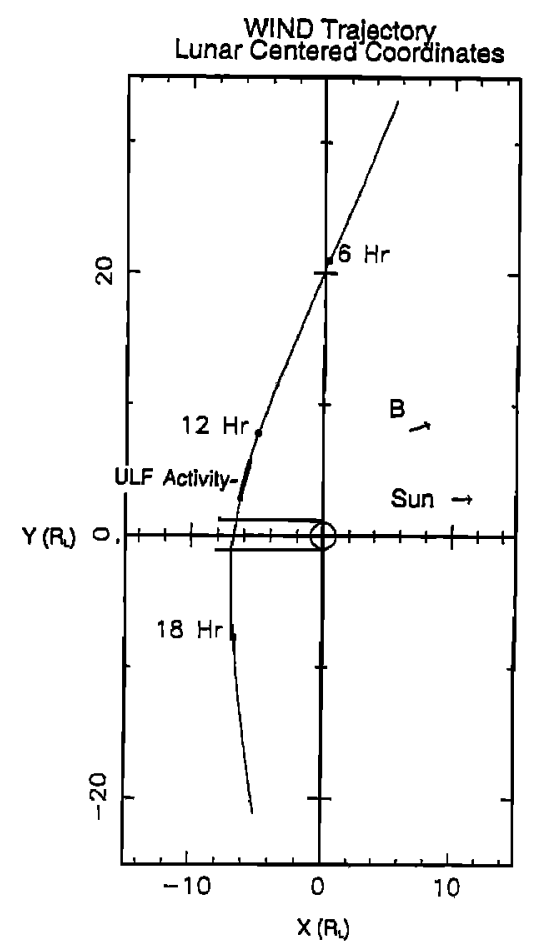

Figure 1. The WIND spacecraft trajectory during the 27 December lunar swingby. The earth is located at $58 \mathrm{R}_{c}$ (or $212 \mathrm{R}_{1}$ ) at a azmuthial phi angle of $144^{\circ}$. The direction is thus towards the upper left hand margin in the figure. 
of the body, making its closest approach on the antisunward side. The moon was located nearly $60 \mathrm{R}_{\varepsilon}$ upstream of the earth near 8 hours local time. Thus, the spacecraft was positioned in the solar wind, upstream and distant from the terrestrial magnetosphere. Owen et al. [1996] show a rather symmetric magnetic disturbance detected by MFI associated with the wake. WIND first entered the penumbra region at 1415 UT, where it remained for approximately 27 minutes. During this period, the measured field magnitudes were depressed relative to nominal values measured by IMP-8 in the unperturbed solar wind. The spacecraft then entered theumbra region between 1442-1523 UT where the field magnitudes rose about $2 \mathrm{n} T$ above nominal levels. From 1523-1540 UT, the spacecraft was again in a weak penumbra region. As described by Owen et al., the magnetic signature of the wake crossing is quite consistent with those made previously by Explorer 35 . The spacecraft was in the lunar shadow (and experienced a substantial reduction in sunlight to the solar cells) between 1458-1532 UT. During the umbra/shadow crossing, the solar wind experiment (SWE) detected a large reduction in the electron density [Ogilvie et al, 1996] and the WAVES radio receivers detected a substantial reduction in the electron plasma frequency [Kellogg et al., 1996], such observations being consistent with the absence of electrons in the wake.

Besides these DC magnetic, particle, and electric wave observations, the WIND magnetometer also detected anomalous ULF magnetic wave activity that occurred continuously for nearly an hour prior to the wake entry. Unlikethesituation with Explorer 35, there is now complete magnetic waveform and simultaneous higl resolution electron measurements which can be used to uniquely identify the physical processes occurring in this active region. The ULF wave activity and corresponding particle measurements are the subject of this work.

\section{Observations}

Figure 1 shows the WIND spacecraft trajectory during the 27 December lunar swingby. The closest approach to the moon occurred near 1448 UT at a distance of about 6.8 lunar radii $\left(R_{Y}\right)$, when the spacecraft was antisunward of the moon. The entry into the lunar wake, as defined by the magnetometer experiment, occurred at $1415 \mathrm{UT}$, and the spacecraft remained in the region for the following 85 minutes. Between 1312 UT and $1325 \mathrm{UT}$, the magnetic field vector swung in phi angle from $0^{\circ}$ to $30^{\circ}$. During this quick swing, the field line connected to the spacecraft became connected to the wake and subsequently, ULF emission commenced. The angle of the field at wave activation was about $15^{\circ}$, when the spacecraft was about $5.5 \mathrm{R}_{1}$ from the wake. This observation suggests the active structures associated with the wake may extend as far as $20 R_{1}$ behind the moon. The field phi angle remained between $20^{\circ}$ and $30^{\circ}$ for the remainder of the of the inbound period, and the theta angle remained near $90^{\circ}$ throughout the period (i.e., nearly in the ecliptic plane). The magnetic field lines connected to the spacecraft missed the terrestrial bow shock by over $20 \mathrm{R}$, thus the spacecraft did not become magneticallyconnected to the terrestrial bow shock during this period.

Figure 2 is a spectrogram displaying the ULF wave activity detected between $21 \mathrm{mHz}$ and $5.43 \mathrm{~Hz}$ by the WIND magnetometer $\mathrm{z}$ - sensor, the sensor oriented along the spacecraft spin axis. In the figure, the vertical axisrepresentsincreasingfrequency, thehorizontalaxisrepresentsincreasing time, and the wave intensity is denoted by the color (blue, least intense; red, most intense). The lunar wake region as defined by the magnetometer is indicated on the spectrogram. The z-sensor data is used for "quick-look" spectral analysis since the spin modulation of the magnetic field near $0.3 \mathrm{~Hz}$ is negligible.

The analysis of the z-sensor data is as follows: Measurements obtained at a rate of 10.87 samples/second were grouped into sets of 500 samples and Fouriertransformed. The power at each of the resulting 250 frequencies between $5.43 \mathrm{~Hz}$ and $21 \mathrm{MHz}$ was calculated. The power at each frequency and time was then plotted on the spectrogram. Figure 2 shows the results from the inboard magnetometer z-sensor. An essentially identical spectrogram was obtained from the outboard $\mathrm{z}$-sensor.

As indicated in Figure 2, prior to 1317 UT, much of the wave activity was broadband Alfvenic turbulence typically observed in the solar wind. However, at 1317 UT, a very unusual narrowband tone was detected between 1-2 Hz that persisted for nearly 60 minutes prior to the wake entry. The tone started initially near $1.2 \mathrm{~Hz}$ at $1317 \mathrm{UT}$, steadily rose in frequency to above $2 \mathrm{~Hz}$ at $1330 \mathrm{UT}$, and then steadily decreased in frequency to near $1.2 \mathrm{~Hz}$ for the last 45 minutes. The emission ceased as the spacecraft entered the penumbra region. The region of observation is illustrated in Figure 1 ,

During this period of time, the ambient magnetic field magnitude was about $7 \mathrm{nT}$, and consequently, the electron and proton cyclotron frequencies were $182 \mathrm{~Hz}$ and $0.1 \mathrm{~Hz}$, respectively. The electron and proton plasma frequencies are $19.3 \mathrm{kHz}$ and $450 \mathrm{~Hz}$, respectively. The observedemission frequency lies wellabovethe proton cyclotron frequency, and thus is tentatively identified as the whistler mode. Further confirmation of the mode is presented below.

Figure 3 shows an individual magnetic field spectrum taken during a three minute period starting from 1345 UT, when the emission was occurring. In this case, measurements from all three sensors in an inertial coordinate system (with spin effects removed) were used to obtain the emission spectral density. Note the presence of a distinct enhancement in the signal level just above $1 \mathrm{~Hz}$, which is the whistler emissions. The signal level of the whistler waves is about $2 \times 10^{-2} \mathrm{nT}^{2} / \mathrm{Hz}$ above the background level. The three lower panels in the figure show the detected magnetic waveforms in the $x, y$, and $z$ sensors for a duration of about 36.8 seconds between the frequencies of $1 \mathrm{~Hz}$ and $2 \mathrm{~Hz}$. Note that a clear and coherent wave near $1 \mathrm{~Hz}$ is present. Nominally, the signal strength of the upstream whistler activity is near $0.1 \mathrm{nT}$, and represents a variation in the field (i.e., Delta-B/B) of about $1 \%$. Compared to the signal levels of the ULF activity observed by Explorer 35 in close to the moon, the WIND observations are weaker by a factor of 20.

\section{Analysis}

Thenarrowbanded whistler waves observed prior to the wake crossing are propagating in the solar wind frame of reference, and thus appear as a Doppler-shifted signal in the observing frame of reference. At ULF frequencies, the Doppler shift can become comparable to the wave frequency, and thus give rise to a substantial shift in wave frequency from its true frequency. However, we are in a very fortuitous situation, since it appears the true wave frequency may be derivable from the observations. The Solar Wind (SWE) experiment can make measurements of the solar wind electron bulk flow speed. During the period of wave activity, there were two major excursions in electron bulk flow, and each excursion had a profound affect on the emission's central frequency. Such an affect is expected in the case of a Doppler-shifted emission. For example, the flow speed increased from a minimum of about $345 \mathrm{~km} / \mathrm{sec}$ at 1317 UT to a maximum of about $447 \mathrm{~km} / \mathrm{sec}$ at $1330 \mathrm{UT}$. The corresponding change in observed wave frequency was $1.20 \mathrm{~Hz}$ (a frequency minimum) to $2.14 \mathrm{~Hz}$ (a frequency maximum) at this same time. The projection of the wave number parallel to the solar wind flow is derivable from the difference in the Doppler-shift equation at the two times, and is

$$
\begin{aligned}
k \cos \left(\theta_{s w}\right) & =-\left(\omega_{1}-\omega_{2}\right) /\left(V_{1}-V_{2}\right) \\
& =-6.0( \pm 1.5) \times 10^{-5} \mathrm{~m}^{-1}
\end{aligned}
$$

The range of values represents the error in the velocity measurement, which possesses some intrinsic spread in values due to the inherent noise in the measurement. Inserting these values back in the Doppler shit equation, one obtains an approximate value of the true whistler wave frequency of $-12.26 \mathrm{rad} / \mathrm{sec}$, or about $-2 \mathrm{~Hz}$. The minus in the wave frequency indicates that the wave is being convected in the downstream direction, with a phase velocity, $v_{p h}$, less than that of the solar wind bulk flow speed, $V>v_{p h}$. However, in order to observe the waves in the upstream region, the wave group velocity must be greater than the solar wind bulk flow speed, $v_{\mathrm{g}}>\mathrm{V}>\mathrm{v}_{\mathrm{ph}}$. Such conditions are possible, and have been observed with narrowband ULF whistler emissions propagating upstream of the terrestrial bow shock [Fairfield, 1974]. A similar $k$ value and true wave frequency was obtained for the second bulk flow increase between 1400 UT and 1415 UT.

Based on the true wave frequency of $2 \mathrm{~Hz}$ and the estimate of the wave vector, a determination of the whistler wave resonant energy is possible. This resonant energy represents the energy of the electrons responsible for generating the observed wave mode. In general, a resonance is required between the electrons and wave in order for energy to be exchanged. The resonance condition between the two is defined as:

$$
\left.\left(\omega-n \omega_{e}\right) / k,=\right)_{\text {res }}
$$

where $v_{\text {|irw }}$ is the parallel electron velocity, $k_{\mid \text {I }}$ is the parallel component of the wave number, and $n$ is an integer. In the cases of $n=+/-1,+/ 2$, etc. the fundamental and harmonic cyclotron resonances defines the interaction between the wave and electrons, while in the case of $n=$ 0 , a Cerenkov resonance is occurring between the waves and the electrons. For this estimate, we assume that $\mathrm{k}_{\|}$has a value of approximately 6.0 $(+/-1.5) \times 10^{-5} \mathrm{~m}^{-1}$ like that derived previously from the variations of the waves with the bulk flow speed. As discussed below, this estimate is quite consistent with the $\mathrm{k}_{\| 1}$ derived from whistler wave analysis 
via cold plasma theory [Stix, 1962]. The electron cyclotron frequency is near $182 \mathrm{~Hz}$. Thus, for a first-order cyclotron resonance $(n=+/-1)$, $E_{r e s}=1 / 2 m_{e} v_{\text {rex }}{ }^{2}$ is between $500-1500 \mathrm{eV}$, while for a Cerenkov resonance $(n=0)$, the resonant energy is between $0.07-0.19 \mathrm{eV}$. The Cerenkov resonant energies are extremely low, and the waves would thus interact with electron of the primary solar wind stream. Such electrons would quickly damp any wave activity, thus the Cerenkov interaction is ruled out as a viable interaction for wave generation.

The resonance energy of the cyclotron interaction can be compared to theSWE electron measurements. Specifically, it is of interest to determine if an unusual electron feature is occurring in association with the advent of the ULF turbulence. Figure 4 shows an overview of the SWE electron measurements during the period the ULF wave activity was observed. Specifically, electron pitch angle spectrograms are shown for 94 and

\section{SPECTRAL ANALYSIS B}

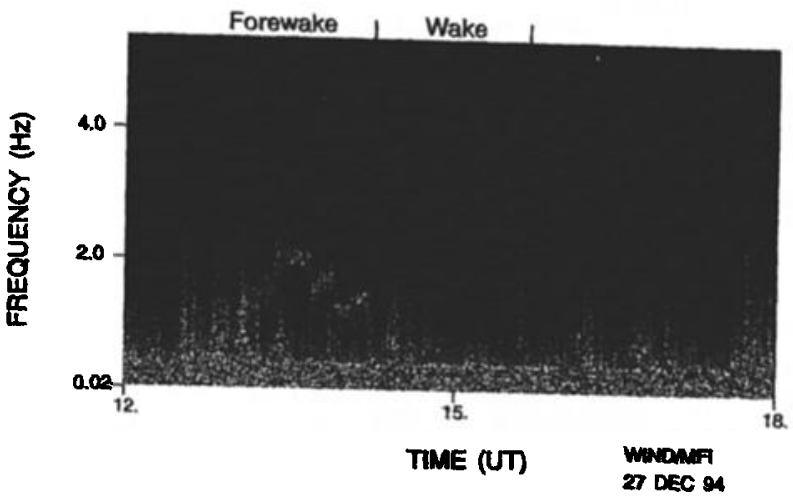

Figure 2. A spectrogram showing the ULF wave intensity from the WND magnetometer (MFI) z-sensor as a function of frequency-versus-time. Red is most intense while blue is least intense.
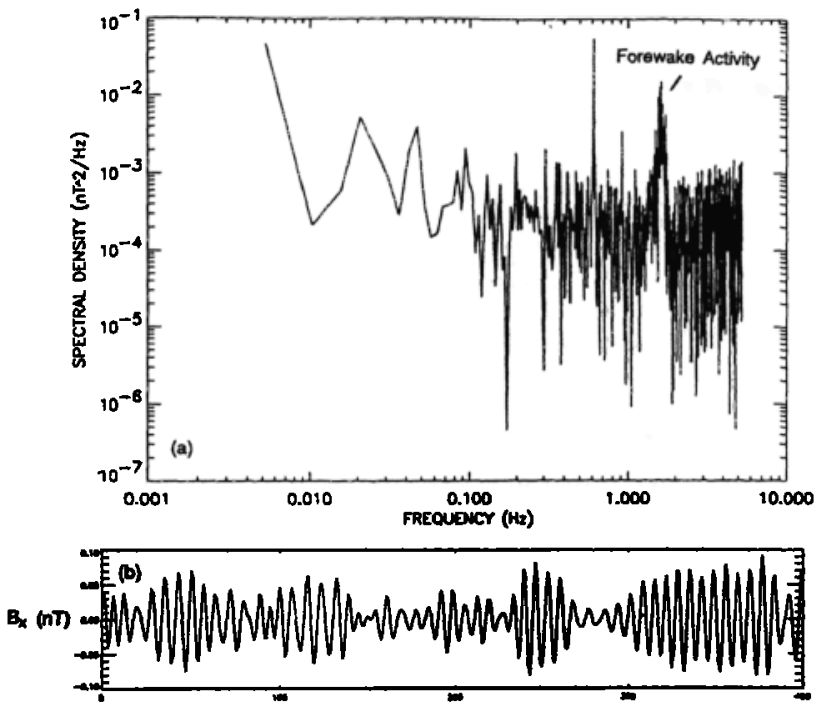

B. (nT)

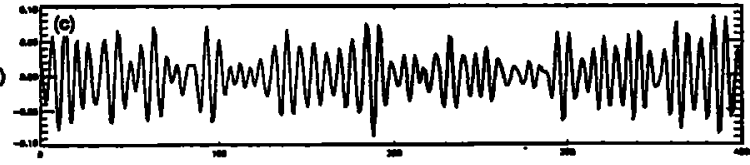

B (nT)

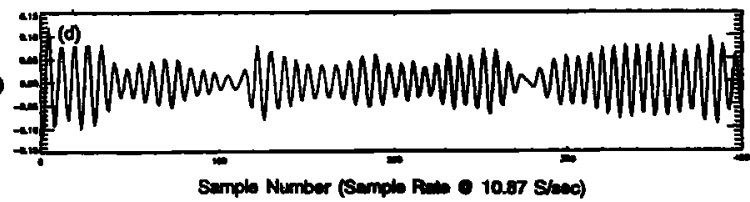

Figure 3. An individual unsmoothed magnetic field spectrum from the WND magnetometer (MFI) for a three minute period near $1345 \mathrm{UT}$. Note the wave enhancement between 1-2 Hz, which is the whistler waves of interest in this note. Waveforms of the whistlers from the three inagntometer sensors in a 36.8 second period between $1-2 \mathrm{~Hz}$ are also shown.
$442 \mathrm{eV}$, along with a sample electron velocity distributions at $1315 \mathrm{UT}$. The spectrograms show electron activity as a function of angle with respect to the local magnetic field. In this presentation, $180^{\circ}$ pitch angle implies flow antiparallel to the magnetic field, in a direction towards the lunar wake. In contrast, $0^{\circ}$ pitch angle implies flow parallel to the magnetic field, in a direction away from the lunar wake. As indicated in the spectrograms, between 1315 and 1415 UT, there is a significant electron component flowing outward from the wake that counterstreams the solar wind. This activity is quite pronounced at $94 \mathrm{eV}$ (as indicated in the figure by the enhanced "yellow" region near $0^{\circ}$ between 1315 and 1415 UT). A counterflowing component also exists at $442 \mathrm{eV}$, having a pitch angle of $30^{\circ}-60^{\circ}$. This higher energy component is of interest since it coincides, in part, with the calculated resonance energy of the ULF waves. This higher energy component could be the driver of the wave activity.

Figure 4 also includes an electron velocity distribution at $1315 \mathrm{UT}$, along with the reduced distribution profile calculated over the same time interval. Note that the central feature is the primary solar wind electrons.

\section{SOLAR WIND ELECTRONS}
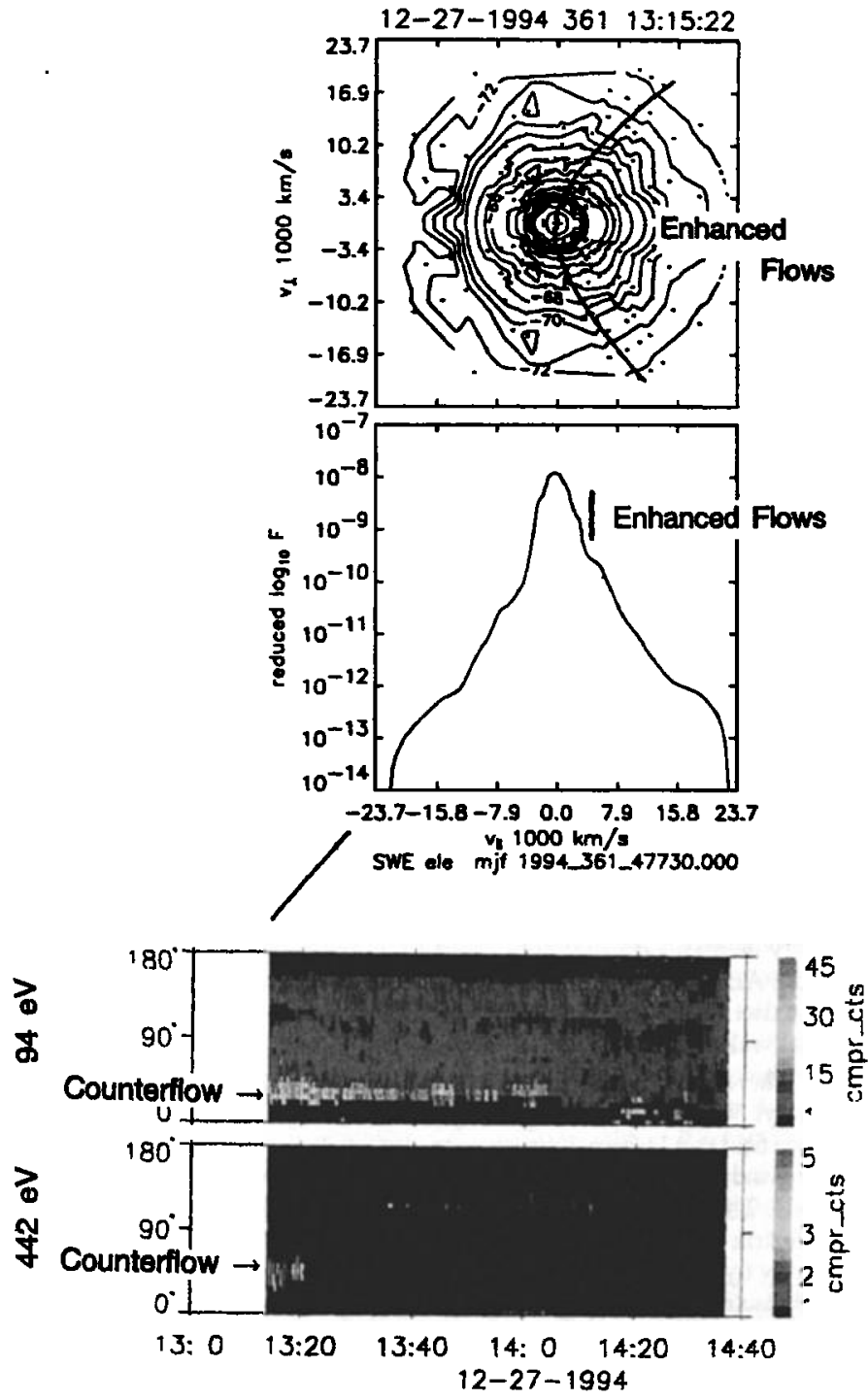

Figure 4. The solar wind electrons as measured by the WIND SWE experiment. Pitch angle spectrograms are shown for $94 \mathrm{eV}$ and 442 eV electrons, along with an individual electron distribution and its associated reduced distribution at 1315 UT. Note in the spectrograms the enhanced electron flows in the counterstreaming direction away from the wake back into the solar wind. Such electron distributions have anisotropies at energies consistent with the whistler cyclotron resonance process, and thus could ultimately drive the observed ULF wave activity. 
However, there is an obvious anomalous electron flow that is propagating counter to the incoming solar wind. This component is indicated in the figures. For example, the distribution indicates the presence of electrons counterflowing from the wake, appearing as a very hot anisotropic beam-type distribution. In the particular example, the anomalous flow appears to have both an obliquely-propagating and parallel propagating component. Theformercomponentappearsas "ears" on the distributions, with enhancements extending to many $100^{\prime}$ 's of electron volts. The latter is identified by the ridges of extended contours in the parallel direction. Such distributions are typically observed simultaneous with the ULF magnetic waves, and could be the free energy source for the waves. We recognize that the presence of electron flows upstream of the lunar wake is reminiscent of the electron flows in the foreshock regions of bow shocks [Filbert and Kellogg, 1979; Fitzenreiter, 1995].

According to cold plasma theory [Stix, 1962], the whistler mode index of refraction, $n$, in the solar wind is derivable as

$$
n^{2}=\omega_{p}^{2} /\left(\omega \omega_{c} \cos \theta-\omega^{2}\right)
$$

Given the conditions adjacent to the wake (electron plasma frequency of $19.3 \mathrm{kHz}$, electron cyclotron frequency of $182 \mathrm{~Hz}$, and wave frequency of $2 \mathrm{~Hz}$ ), the wave number, $\mathrm{k}$, for a wave propagating exactly parallel to the magnetic field (i.e., normal angle of $0^{\circ}$ ) is $\mathrm{k}=4.2 \times 10^{-3} \mathrm{~m}^{-1}$. For oblique propagation, cold plasma theory indicates that $\mathrm{k}$ increases steadily but slowly from $4.2 \times 10^{-5} \mathrm{~m}^{-1}$ at $0^{\circ}$ normal angle, to $4.5 \times 10^{-5} \mathrm{~m}^{-1}$ at $30^{\circ}$, $6.0 \times 10^{-5} \mathrm{~m}^{-1}$ at $60^{\circ}$, and to $8.4 \times 10^{-5} \mathrm{~m}^{-1}$ at $75^{\circ}$. Thus, our measurement of $k$ obtained directly from comparing the emission frequency with solar wind bulk flow speed is quite consistent with the theoretical values obtained from cold plasma theory. The value of $k$ measured is $6.0(t /-1.5) \mathrm{x}$ $10^{-5} \mathrm{~m}^{-1}$ and is slightly above the theoretical parallel propagating value. According to cold plasina theory, the observed $\mathrm{k}$ is consistent with a wave propagating at a normal angle oriented at $>30^{\circ}$ relative to the local magnetic field.

Given the conditions upstream of the wake, the maximum theoretical value of the phase velocity, $v_{p h}=c / n$, is $300 \mathrm{~km} / \mathrm{sec}$, and occurs for a wave normal angle of $0^{\circ}$. All other angles have lower phase velocities. In contrast, the group velocity, $v_{g}$, (as defined by Eq. (3) of Fairfield [1974]) remains large, with a minimum value of $v_{g}$ never extending below $535 \mathrm{~km} / \mathrm{second}$. Comparing these characteristic wave velocities to the solar wind electron bulk flow speed, V, (which remains between 345 and $460 \mathrm{~km} / \mathrm{sec}$ during the active period), we find that $v_{g}>V>$ $V_{\mathrm{ph}}$. This result is self-consistent with the observations of a downstream convected wave discussed previously, which when Doppler shifted possessed a "negative" frequency. Even though the wave normal angle associated with the observed $\mathrm{k}$ is oblique, exceeding $30^{\circ}$, the corresponding group velocity vector is oriented close to the magnetic field line, extending no more than $19^{\circ}$. Thus, the emissions ray path is aligned relatively close to the magnetic field, in an upstream orientation, while the wave phase is convected at a more oblique angles with respect to the magnetic field.

\section{Interpretation}

Based on the WIND measurements, it appears that electromagnetic activity increases on magnetic field lines threading through the wake region. Anisotropic electron populations in the counterstreaming direction also exists on the field lines. This activity appears as a precursor to the actual wake region.

There are a couple of possible scenarios for generating this upstream activity. Within the wake, it is expected that an ambipolar quasi-DC electric field should form due to the violation of charge neutrality. Specifically, solar wind electrons quickly fill in behind the wake in a region devoid of ions. This occupation of one charged species over the other will create an electric field which repels electrons. The situation has been described recently by Ogilvie et al. [1996]. This field should reflect incoming solar wind electrons and may give rise to the enhanced electron flows away from the wake region, thereby creating an electron anisotropy and subsequent ULF wave activity via stimulated emission (i.e., an instability).

A second viable scenario is that electrons become concentrated in pitch angle forming a "source cone" due to the movement from the relatively higher magnetic field region in the wake to lower fields in adjacent regions outside. It should be noted that electrons within the wake near $90^{\circ}$ pitch angle will be concentrated to near $60^{\circ}$ pitch angle in regions outside the wake in association with the $25 \%$ decrease in field strength. The formation of such a source cone distribution is consistent with the measured distribution shown in Figure 4. Again, this source cone is anisotropic and may be capable of generating ULF emissions.

Anisotropic distributions are also present upstream of the bow shock, forming what is now commonly called the foreshock region. Such flows are believed to be the source of both ULF [Fairfield, 1974; Hoppe and Russell, 1980] and VLF [Filbert and Kellogg, 1979] wave activity. This preposition concerning ULF whistler waves was recently reanalyzed by Orlowski et al., [1995] and Wong and Smith [1995].

By analogy, one might also consider the region upstream of the wake as a "forewake", implying the presence of anisotropic electron distributions and wave activity. This precursor region appears to convey information to the inflowing solar wind electrons on the upcoming presence of the lunar wake. Future WIND passages through the lunar nightside region should yield valuable insights on the formation of the wake and precursor regions.

\section{References}

Fairfield, D. H., Whistler waves observed upstream from collisionless shocks, J. Geophys. Res., 79, 1368, 1974.

Filbert, P. C., and P. J. Kellogg, Electrostatic noise at the plasma frequency beyond the earth's bow shock, J. Geophys. Res., 84, 1369, 1979.

Fitzenreiter, R. J. , The electron foreshock, Adv. Space Sci., 15, (8/9)9 1995.

Fitzenreiter, R. J., J. D. Scudder, and A. J. Klimas, Three dimensional analytical model of the spatial variations of the foreshock electron distributon function: Systematics and comparisons with ISEE observations, J. Geophys. Res., 95, 4155,1990 .

Hoppe, M., and C. T. Russell. Whistler mode wave packets in the earth's foreshock region, Nature, 287, 417, 1980.

Krall, N. A., and D. A. Tidman, Magnetic field fluctuations near the moon, J. Geophys. Res., 74, 6439, 1969.

Kellogg, P. J., K. Goetz, S. J. Monson, J.-L. Bougeret, R. Manning, and M. L. Kaiser, Waves on the moon's wake, Geophys.Res. Lett., this issue, 1996.

Ness, N. F. , and K. H. Schatten, Detaction of interplanetary magnetic field fluctuations stimulated by the lunar wake, J. Geophys. Res., 74, 6425, 1969.

Ness, N. F., Interaction of the soalr wind with the moon, in Solar Terrestrial Physics, part II, 159, D. Reidel Pub. Co. Holland, 1970.

Owen, C. J., R. P. Lepping, K. Ogilvie, J. A. Slavin, W. Farrell, and J, B. Bymes, Thelungrwakeat $6.8 R_{1}$ : WTNDmagnetic field observations, Geophys.Res. Lett., this issue, 1996.

Ogilvie, K. W., J. T. Steinberg, R. J. Fitzenreiter, C. J. Owen, A. J. Lazarus, W. M. Farrell, and R. B. Tolbert, Observations of the lunar plasma wake from the WND spacecraft on December 27 1994. Geophys. Res. Lett., this issue, 1996.

Orlowski, D. S., C., T. Russell, Comments on "Electron beam excitation of upstream waves in the whistler mode frequency range" by $H$. K. Wong and C. W. Smith, J. Geophys. Res., 100, 17139, 1995.

Stix, T. H. , Theory of plamsa waves, McGraw-Hill, New York, 1962.

Whang, Y.-C., and N. F. Ness, Observations and interpretation of the lunar Mach cone, J. Geophys. Res., 75, 6002, 1970.

Wong, H. K., and C. W. Smith, Reply, J. Geophys. Res., 100, 17141, 1995.

J. B. Byrnes, W. M. Farrell, R. J. Fitzenreiter, R. P. Lepping, and K. W. Ogilvie, Laborntory for Extraterrestrial Physics, NASA/Goddard SFC, Greenbelt, MD 20771

C. J. Owen, now at Astronomy Unit, School of Mathematical Sciences, Queen Mary and Westfield College, Mile End Road, London, E1 4NS, UK

F. Neubauer, Institut fur Geophysics und Meteorlogie der Universitat zu Koln, 500 Koln-41, Germany

Received: October 23, 1995; revised: March 8, 1996; accepted: March 19, 1996) 